\title{
First-principles study of two- and one-dimensional honeycomb structures of boron nitride
}

\author{
M. Topsakal, ${ }^{1}$ E. Aktürk, ${ }^{1}$ and S. Ciraci ${ }^{1,2, *}$ \\ ${ }^{1}$ UNAM-Institute of Materials Science and Nanotechnology, Bilkent University, Ankara 06800, Turkey \\ ${ }^{2}$ Department of Physics, Bilkent University, Ankara 06800, Turkey
}

(Received 22 December 2008; revised manuscript received 12 February 2009; published 30 March 2009)

\begin{abstract}
This paper presents a systematic study of two- and one-dimensional honeycomb structures of boron nitride (BN) using first-principles plane-wave method. In order to reveal dimensionality effects, a brief study of all allotropic forms of three-dimensional (3D) BN crystals and truly one-dimensional atomic BN chains are also included. Two-dimensional (2D) graphenelike $\mathrm{BN}$ is a wide band-gap semiconductor with ionic bonding through significant charge transfer from B to N. Phonon-dispersion curves demonstrate the stability of 2D BN flakes. Quasi-one-dimensional (1D) armchair BN nanoribbons are nonmagnetic semiconductors with edge states. Upon passivation of $\mathrm{B}$ and $\mathrm{N}$ with hydrogen atoms these edge states disappear and the band gap increases. Bare zigzag BN nanoribbons are metallic but become a ferromagnetic semiconductor when both their edges are passivated with hydrogen. However, their magnetic ground state, electronic band structure, and band gap are found to be strongly dependent on whether B or N edge of the ribbon is saturated with hydrogen. Vacancy defects in armchair and zigzag nanoribbons affect also the magnetic state and electronic structure. Harmonic, anharmonic, and plastic regions are deduced in the variation in the total energy of armchair and zigzag nanoribbons as a function of strain. The calculated force constants display a Hookian behavior. In the plastic region the nanoribbon is stretched, whereby the honeycomb structure of hexagons change into different polygons through sequential structural transformations. In order to reveal dimensionality effects these properties are contrasted with those of various 3D BN crystals and 1D BN atomic chain.
\end{abstract}

DOI: 10.1103/PhysRevB.79.115442

PACS number(s): 81.05.Uw, 73.22.-f, 75.75.+a, 63.22. $-\mathrm{m}$

\section{INTRODUCTION}

Synthesis of a single atomic plane of graphite, i.e., graphene with covalently bonded honeycomb lattice, has been a breakthrough for several reasons. ${ }^{1-3}$ First, electrons behaving as if massless Dirac fermions have made the observation of several relativistic effects possible. Second, stable graphene has disproved previous theories, which were concluded that two-dimensional (2D) structures cannot be stable. Graphene displaying exceptional properties, such as high mobility even at room temperature, ambipolar effect, Klein tunneling, anomalous quantum hall effect, etc., seems to offer novel applications in various fields. ${ }^{4}$ Not only 2D graphene but also its quasi-one-dimensional (1D) forms, such as armchair and zigzag nanoribbons, have shown novel electronic and magnetic properties, ${ }^{5-7}$ which can lead to important applications in nanotechnology. As a result, 2D honeycomb structures derived from group IV elements and group III-V and II-VI compounds are currently generating significant interest owing to their unique properties.

Boron nitride $(\mathrm{BN})$ in ionic honeycomb lattice which is the group III-V analog of graphene has also been produced having desired insulator characteristics. ${ }^{8}$ Nanosheets, ${ }^{9,10}$ nanocones, ${ }^{11}$ nanotubes, ${ }^{12}$ nanohorns, ${ }^{13}$ nanorods, ${ }^{14}$ and nanowires ${ }^{15}$ of $\mathrm{BN}$ have already been synthesized, and these systems might hold promise for novel technological applications. Among all these different structures, BN nanoribbons (BNNRs), where the charge carriers are confined in two dimensions and free to move in the third direction, are particularly important due to their well-defined geometry and possible ease of manipulation.

$\mathrm{BN}$ nanoribbons posses different electronic and magnetic properties depending on their size and edge termination. Recently, the variation in band gaps of BN nanoribbons with their widths and Stark effect due to applied electric field have been studied. ${ }^{16,17}$ Recently the magnetic properties of zigzag BN nanoribbons (ZBNNRs) have been investigated. ${ }^{18}$ Halfmetallic properties have been revealed from these studies which might be important for spintronic applications. Production of graphene nanoribbons as small as $10 \mathrm{~nm}$ in width has been achieved ${ }^{19,20}$ and similar techniques are expected to be developed for $\mathrm{BN}$ nanoribbons.

A thorough understanding of $2 \mathrm{D} \mathrm{BN}$ honeycomb structure and their various nanoribbons is important for further study of this graphenelike compounds. BN by itself provides very interesting chemical and physical properties, which may lead to important applications. In this paper, we present a detailed $a b$ initio study of electronic, magnetic, and elastic properties of 2D (graphenelike) BN and bare and hydrogen-passivated quasi-1D BNNRs. We also investigated the effect of the vacancy defects on these properties. To reveal the dimensionality effects we include also a short discussion regarding three-dimensional (3D) BN bulk crystals and 1D BN atomic chains. We found that $2 \mathrm{D} \mathrm{BN}$ is a nonmagnetic wide bandgap semiconductor. The ionic bonding due to a significant amount of charge from $\mathrm{B}$ to $\mathrm{N}$ atom opens a gap and hence dominates electronic structure. Calculated phonon-dispersion curves provide a clear evidence that 2D BN flakes are stable. The armchair and zigzag nanoribbons of $\mathrm{BN}$ display even more interesting electronic and magnetic properties. Bare and hydrogen-passivated armchair $\mathrm{BN}$ nanoribbons (ABNNRs) are nonmagnetic wide band-gap semiconductor. The value of the band gap of ABNNR having a width of $w$ $>10 \AA$ is practically independent from the width of nanoribbons. While the bare ZBNNRs are ferromagnetic metal, they become nonmagnetic semiconductor upon the passivation of both edges. We found that $2 \mathrm{D} \mathrm{BN}$ and its nanoribbons have properties that are complementary to graphene. 


\section{MODEL AND METHODOLOGY}

We have performed first-principles plane-wave calculations within density-functional theory (DFT) using projector augmented wave (PAW) potentials. ${ }^{21}$ The exchangecorrelation potential has been approximated by generalized gradient approximation (GGA) using the PW91 (Ref. 22) functional both for spin-polarized and spin-unpolarized cases. All structures have been treated within supercell geometry using the periodic boundary conditions. A planewave basis set with kinetic-energy cutoff of $500 \mathrm{eV}$ has been used. In the self-consistent potential and total-energy calculations the Brillouin zone (BZ) is sampled by special $\mathbf{k}$ points. The numbers of these $\mathbf{k}$ points are $(15 \times 15 \times 15)$ for bulk BN, $(25 \times 25 \times 1)$ for $2 \mathrm{D} \mathrm{BN}$, and $(25 \times 1 \times 1)$ for nanoribbons, respectively, and are scaled according to the size of superlattices. All atomic positions and lattice constants are optimized by using the conjugate gradient method, where the total energy and atomic forces are minimized. The convergence for energy is chosen as $10^{-5} \mathrm{eV}$ between two steps, and the maximum Hellmann-Feynman forces acting on each atom is less than $0.02 \mathrm{eV} / \AA$ upon ionic relaxation. A large spacing of $\sim 10 \AA$ between monolayers and $\sim 13 \AA$ between edges of two ribbons in adjacent supercells have been taken to prevent interactions between them. The pseudopotentials having three and five valence electrons for the B (B: $\left.2 s^{2} 2 p^{1}\right)$ and $\mathrm{N}$ ions (N: $\left.2 s^{2} 2 p^{3}\right)$ were used. Numerical calculations have been performed by using the VASP package. ${ }^{23,24}$ The phonon-dispersion curves are calculated within densityfunctional perturbation theory (DFPT) using plane-wave methods as implemented in the PWSCF software..$^{25}$

\section{3D BN CRYSTALS AND 1D ATOMIC CHAIN}

In this section, we present our theoretical calculations on 3D bulk BN crystals and truly 1D BN atomic chain. Earlier these 3D bulk crystals ${ }^{26-30}$ and $1 \mathrm{D}$ atomic chains ${ }^{31}$ have been studied theoretically by using different methods. Our purpose in including these crystals of BN in different dimensionalities is to contrast their properties with those of $2 \mathrm{D}$ and quasi-1D honeycomb structures of $\mathrm{BN}$ and also reveal dimensionality effects.

\section{A. 3D bulk BN crystals}

Three-dimensional bulk crystals include hexagonallayered $\mathrm{BN}$ (h-BN), wurtzite $\mathrm{BN}$ (wz-BN), and zinc-blende BN (zb-BN) structures. Their atomic configurations and primitive unit cells are described in Fig. 1. By using the expression

$$
E_{C}=E[\mathrm{BN}]-E[\mathrm{~B}]-E[\mathrm{~N}],
$$

where $E[\mathrm{BN}]$ is the total energy per B-N pair of the optimized structure of $\mathrm{BN}$ crystal and $E[\mathrm{~B}]$ and $E[\mathrm{~N}]$ are the total energies of free $\mathrm{B}$ and $\mathrm{N}$ atoms, we calculated the equilibrium cohesive energies of h-BN, wz-BN, and zb-BN crystals as $-17.65,-17.45$, and $-17.49 \mathrm{eV}$ per $\mathrm{B}-\mathrm{N}$ pair, respectively. Accordingly, h-BN, which is the analog of graphite, is the most energetic bulk structure. On the other hand, the cubic BN structure is known to be the second hardest material of all.

The lattice constants of the optimized structures in equilibrium are $a=2.511 \AA, c / a=2.66$, and the distance between the nearest $\mathrm{B}$ and $\mathrm{N}$ atoms is $d=1.450 \AA$ for h-BN layered crystal. For wz BN, optimized values of $a, c / a$, and $d$ are calculated to be $2.542 \AA, 1.64$, and $1.561 \AA$, respectively. The zinc-blende structure has a lattice constant $a=2.561 \AA$ and $d=1.568 \AA$. All our results related with the structural parameters are in good agreement with the experimental and theoretical values ${ }^{26-29,32}$ within the average error of $\sim 1 \%$ (Table I).

The calculated electronic band structure and total and partial (or orbital projected) density of states (DOS) of 3D crystals are presented in Fig. 1. These h-BN, wz-BN, and zb-BN crystals are indirect band-gap semiconductors with calculated band gaps being $E_{G}=4.47,5.72$, and $4.50 \mathrm{eV}$, respectively. The calculated values of $E_{G}$ differ from the earlier ones depending on the method used. ${ }^{33}$ For h-BN having 2D $\mathrm{BN}$ atomic layers in the $(x, y)$ plane, the band structure is composed from the band structures of these individual atomic layers with hexagonal symmetry, which are slightly split due to weak coupling between them. The highest valence band has $\mathrm{N}-p_{z}$ character; the states of the lowest conduction band is formed from B- $p_{z}$ orbitals (the $z$ direction corresponds to " $c$ " in Fig. 1). The overall features of the total density of states (TDOS) are similar for three 3D crystal structures. Valence band consists of two parts separated by a wide intraband gap. The lower part at $\sim-20 \mathrm{eV}$ is projected mainly to N-s and partly to N-p and B- $s$ orbitals. The upper part is due to mainly $\mathrm{N}-p$ and partly B- $p$ orbitals and has similarities in both zb-BN and wz-BN crystals. As for the lower part of the conduction band it is derived mainly from B- $p$ orbitals. The differences of three 3D crystals are pronounced in the lower part of the conduction band.

We calculate the amount of charge on constituent $\mathrm{B}$ and $\mathrm{N}$ atoms in 3D crystals by performing the Löwdin ${ }^{34}$ analysis in terms of the projection of plane waves into atomic orbitals. By subtracting the valencies of free $\mathrm{B}$ and $\mathrm{N}$ atoms from the calculated charge values on the same atoms in 3D crystals, we obtain the charge transfer, $\Delta Q$. The calculated values of $\Delta Q$ for $\mathrm{h}-\mathrm{BN}$, wz BN, and zb $\mathrm{BN}$ are $0.416,0.342$, and 0.334 electrons, respectively. The $\Delta Q$ of $\mathrm{zb} \mathrm{BN}$ and $\mathrm{wz} \mathrm{BN}$ have almost equal values but the $\Delta Q$ of h-BN crystal is significantly larger related to the shorter B-N bond length in h-BN crystal.

\section{B. 1D BN atomic chain}

$\mathrm{BN}$ forms stable segments of linear atomic chain ${ }^{31}$ such as carbon. ${ }^{35}$ This situation is in contrast to the second and third row elements (such as $\mathrm{Si}$ and $\mathrm{Ge}$ ) and III-V compounds and metals (such as $\mathrm{Al}, \mathrm{Au}$, etc.) which can form stable zigzag chain structures instead of linear-chain structures. Our results on optimized chain structure yield the cohesive energy $E_{C}$ $=16.04 \mathrm{eV}$ per B-N pair, the B-N distance $d=1.307 \AA$, the indirect band gap $E_{G}=3.99 \mathrm{eV}$, and charge transfer from B to $\mathrm{N}, \Delta Q=0.511$ electrons. Hence the double bond between $\mathrm{B}$ and $\mathrm{N}$ is ionic. 

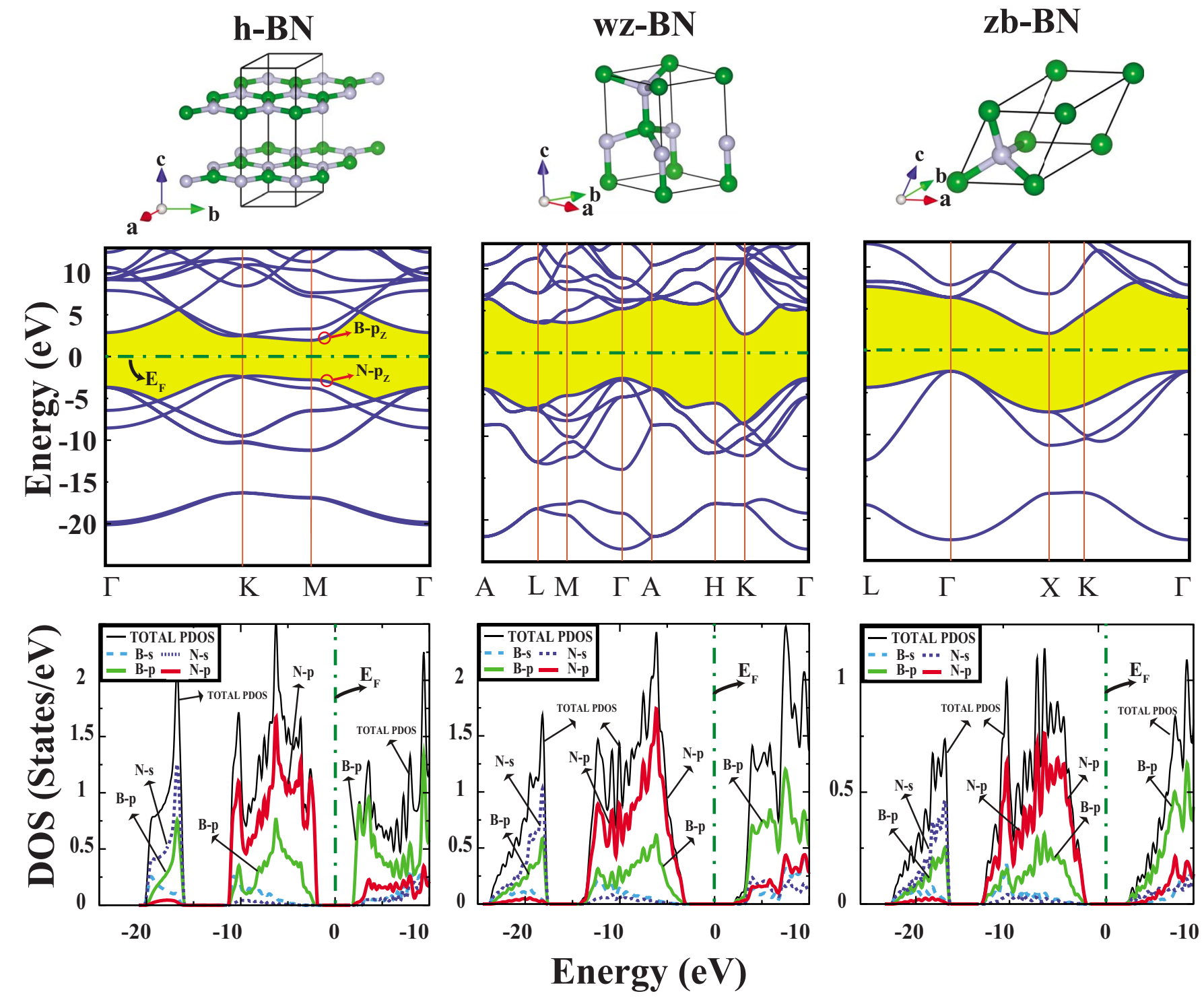

FIG. 1. (Color online) Optimized atomic structure, energy bands, total (TDOS), and orbital projected density of states (PDOS) of various $3 \mathrm{D}$ crystals of $\mathrm{BN}$. (a) Hexagonal (h-BN) whose $\mathrm{B}(\mathrm{N})$ atoms are on top of the $\mathrm{N}(\mathrm{B})$ atoms in the consecutive layer; (b) wurtzite (wz-BN); and (c) zinc-blende (zb-BN) crystals. Dark-green and light-gray balls represent $\mathrm{B}$ and $\mathrm{N}$ atoms, respectively. The band gaps between conduction and valence bands are highlighted. The orbital character of states is indicated for the conduction and valence band edges. The zero of energy is set to the Fermi energy $E_{F}$. All structures are fully optimized.

\section{2D HONEYCOMB STRUCTURE OF BN}

Having discussed the overall structural and elastic properties of 3D and 1D BNs, we now consider 2D BN with hexagonal symmetry. The atomic structure of $2 \mathrm{D} \mathrm{BN}$ is similar to the honeycomb structure of graphene, except that the constituent atoms of the former are from III and V columns

TABLE I. Values of the bond length $d$ in $\AA$, cohesive energy $E_{C}$ in $\mathrm{eV}$ per B-N pair, band gap $E_{G}$ in $\mathrm{eV}$, charge transfer from $\mathrm{B}$ to $\mathrm{N}(\Delta Q)$ in electrons, and lattice constants $(a$ and $c)$ in $\AA$ calculated for various allotropic forms of $\mathrm{BN}$ in different dimensionality.

\begin{tabular}{lccccc}
\hline \hline & $d$ & $E_{C}$ & $E_{G}$ & $\Delta Q$ & Lattice \\
\hline 1D Chain & 1.307 & -16.04 & 3.99 & 0.511 & $a=2.614$ \\
2D BN & 1.452 & -17.65 & 4.64 & 0.429 & $a=2.511$ \\
h-BN & 1.450 & -17.65 & 4.47 & 0.416 & $a=2.511, c / a=2.66$ \\
Wurtzite & 1.561 & -17.45 & 5.726 & 0.342 & $a=2.542, c / a=1.63$ \\
Zinc blende & 1.568 & -17.49 & 4.50 & 0.334 & $a=2.561$ \\
\hline \hline
\end{tabular}


(a)
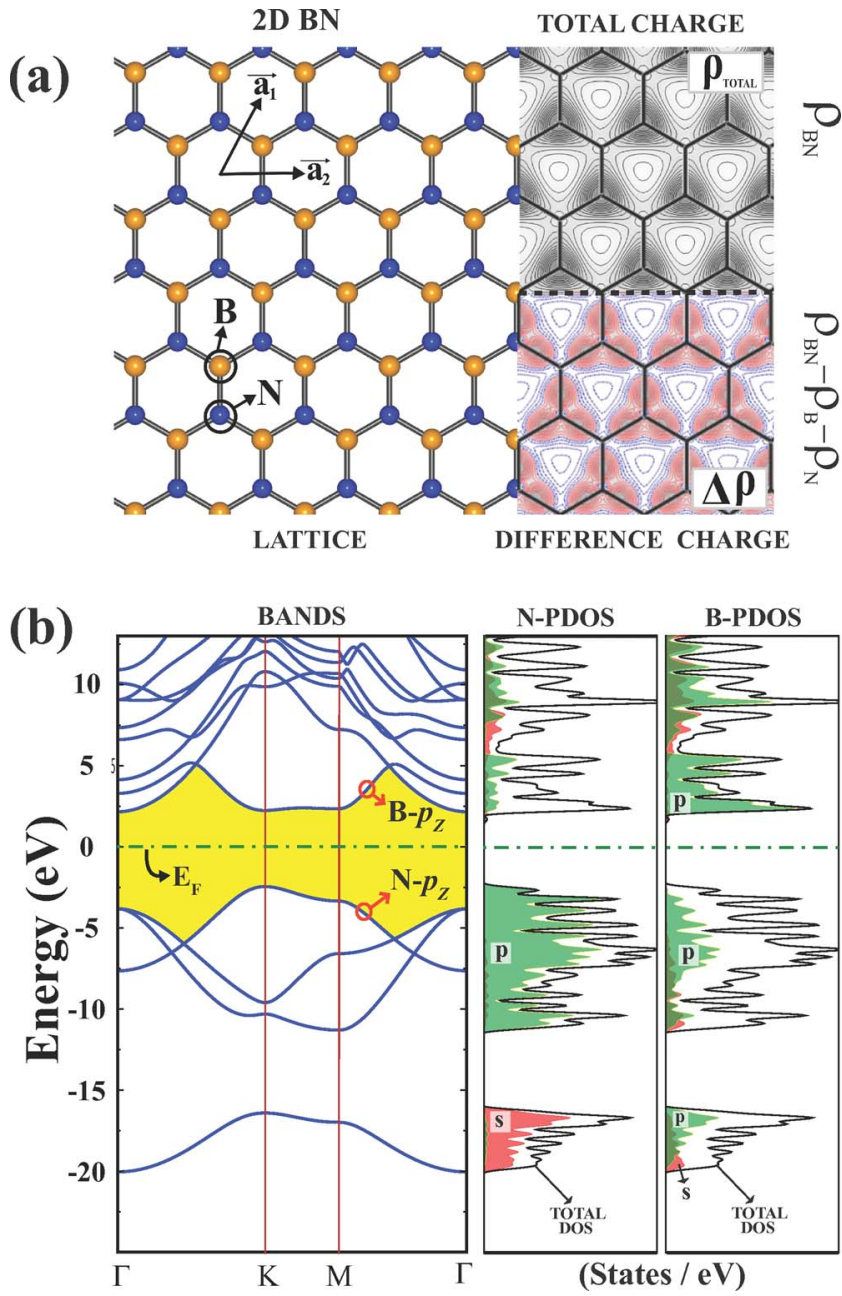

FIG. 2. (Color online) (a) Primitive unit cell of the honeycomb structure of 2D BN together with Bravais lattice vectors. Calculated total charge density $\rho_{\mathrm{BN}}$ and difference charge density $\Delta \rho$ are also shown in the same panel. (b) Calculated electronic structure of $2 \mathrm{D}$ $\mathrm{BN}$ honeycomb crystal together with total, TDOS, and partial density of states, PDOS, on B and $\mathrm{N}$ atoms. The orbital character of the states is also indicated.

of the Periodic Table. Normally, the bond between nearest B and $\mathrm{N}$ atoms is formed from the bonding combination of $\mathrm{B}-s p^{2}$ and $\mathrm{N}-s p^{2}$ orbitals. However, owing to the electronegativity difference between $\mathrm{B}$ and $\mathrm{N}$ atoms, electrons are transferred from B to N. As a result, in contrast to purely covalent bond in graphene the bonding between $\mathrm{B}$ and $\mathrm{N}$ gains an ionic character. The charge transfer from $\mathrm{B}$ to $\mathrm{N}$ dominates several properties of 2D BN including the opening of the band gap. In this respect the $\mathrm{BN}$ honeycomb structure is complementary to graphene.

\section{A. Charge-density analysis and electronic structure}

The atomic structure, atomic charge, charge transfer from $\mathrm{B}$ to $\mathrm{N}$, and the electronic structure of $2 \mathrm{D} \mathrm{BN}$ are presented in Fig. 2. Contour plots of total charge indicate high density around $\mathrm{N}$ atoms. The difference charge density is calculated by subtracting charge densities of free $\mathrm{B}$ and $\mathrm{N}$ atoms from the charge density of $2 \mathrm{D}$ BN, i.e., $\Delta \rho=\rho_{\mathrm{BN}}-\rho_{\mathrm{B}}-\rho_{\mathrm{N}}$. Highdensity contour plots around $\mathrm{N}$ atoms protruding toward the $\mathrm{B}-\mathrm{N}$ bonds indicate charge transfer from $\mathrm{B}$ to $\mathrm{N}$ atoms. This way the B-N bonds achieve an ionic character. The amount of transfer of charge is calculated by Löwdin analysis to be $\Delta Q=0.429$ electrons. Interestingly, $\Delta Q$ is slightly larger than that calculated for $\mathrm{h}-\mathrm{BN}$ but significantly larger than those calculated for wz-BN and zb-BN crystals.

$2 \mathrm{D} \mathrm{BN}$ is a semiconductor. Calculated electronic energy bands are similar to those calculated for h-BN crystal. The $\pi$ and $\pi^{*}$ bands of graphene which cross at the $K$ and $K^{*}$ points of the BZ open a gap in the $2 \mathrm{D} \mathrm{BN}$ as bonding and antibonding combinations of $\mathrm{N}-p_{z}$ and $\mathrm{B}-p_{z}$ orbitals. The contribution of $\mathrm{N}-p_{z}$ is pronounced for the filled band at the edge of valence band. The calculated band gap is indirect and $E_{G}$ $=4.64 \mathrm{eV}$. TDOS and partial density of states show also similarity to those of the h-BN layered crystal presented in Fig. 1.

\section{B. Phonon spectrum}

Even if the structure optimization resulting in the honeycomb structure in Fig. 2 can be taken as an indication for the stability of 2D BN, calculation of phonon-dispersion curves through the diagonalization of dynamical matrix provides a more stringent test for stability. One of the acoustical branches for $\Gamma$ to $K$ curves taking a negative value even at a small region of $\mathrm{BZ}$ indicates the instability of the structure. There have been a number of experimental ${ }^{36}$ and theoretical studies of phonon spectrum of 2D (Ref. 37) and 3D honeycomb BN. ${ }^{38-42}$ Here, the phonon-dispersion curves of h-BN, 2D BN, and 1D BN chain and density of states together with the infrared (IR) and Raman (R) active modes of 2D BN and h-BN at $\Gamma$ point have been calculated by using DFPT as implemented in the PWSCF software. ${ }^{25}$ For the DFPT phonon calculation of bulk h-BN, we used a four-atom primitive cell, which yield 12 phonon branches at the center of BZ in Fig. 3 (a). The symmetry point group is calculated as $D_{6 h}$ (space group $P 6 / \mathrm{mmm}$ ). The irreducible representations at $\Gamma$ is $2 E_{2 g}+2 B_{2 g}+2 A_{2 u}+2 E_{1 u}$. While the modes $E_{1 u}$ and $E_{2 g}$ are doubly degenerate, $B_{2 g}$ and $A_{2 u}$ are nondegenerate. The modes $E_{1 u}$ and $A_{2 u}$ are IR active, the $E_{2 g}$ is Raman active. $B_{2 g}$ is an inactive mode. Our results are in agreement with the previously calculated and experimental data but differ slightly from those of Serrano et al. ${ }^{40}$ While the present GGA calculations predict the $B_{2 g}$ mode as an inactive mode, local-density approximation (LDA) calculations by Serrano et al. found $B_{1 g}$ as an inactive mode. Most of the phonon bands of h-BN are degenerate. This indicates that the coupling between BN layers in h-BN is weak. However, it is well known that the $\mathrm{BN}$ is a polar material with long-range dipole-dipole interaction. This gives rise to the splitting between longitudinal optical (LO) and transverse optical (TO) modes at the $\Gamma$ point. The lowest transverse acoustical (TA) mode has parabolic dispersion as $\mathbf{k} \rightarrow 0$, owing to rapidly decaying interatomic forces for transversal displacements. ${ }^{43}$ Another feature is the overlap of the lowest transversal optical mode with the acoustical modes.

In Fig. 3(b) we show the phonon-dispersion curve of $\mathrm{BN}$ atomic chain. Two TA modes have low frequency and get 
(a)

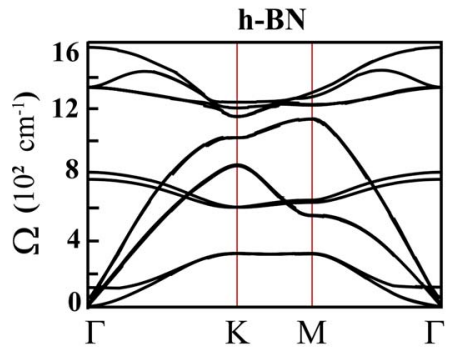

(c)

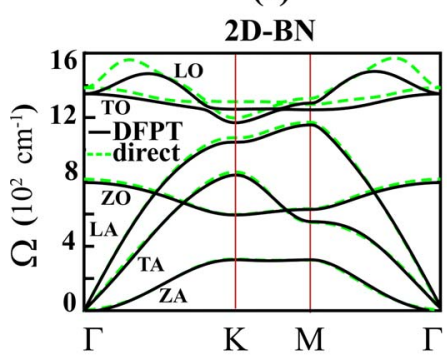

(b)

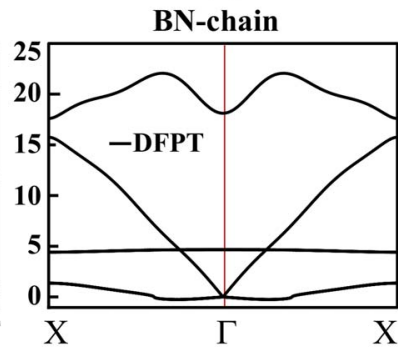

(d)

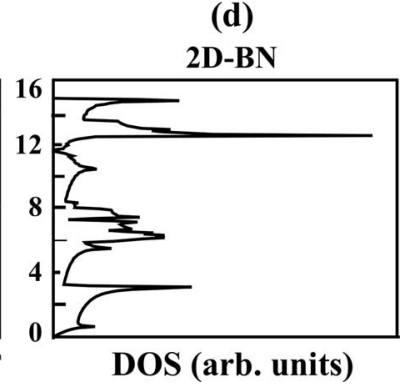

FIG. 3. (Color online) Calculated phonon frequencies versus $\mathbf{k}$ vectors. (a) h-BN crystal. (b) $1 \mathrm{D} \mathrm{BN}$ atomic chain. (c) $2 \mathrm{D} \mathrm{BN}$ honeycomb structure. Phonon modes calculated by force-constant direct method are shown by the green-dashed curve. (d) Density of phonon frequencies (DOS) for the 2D BN honeycomb structure.

very small but negative values near the zone center. This indicates structural instability as $\lambda \rightarrow \infty$. However, the linear segments of BN atomic chain can be stable. Similar to h-BN, the doubly-degenerate TO branch overlaps with the LO branch.

For 2D BN honeycomb structure, the unit cell consists of two atoms. Accordingly, there are three acoustical and three optical branches in Fig. 3(c). The symmetry point group is $D_{3 h}$ [space group $\left.(P-62 m)\right]$. Optical phonon modes at the $\Gamma$ point is given by $A_{2}^{\prime \prime}+2 E^{\prime}$. The mode $A_{2}^{\prime \prime}$ is IR active and the $E^{\prime}$ mode is both IR and Raman active. The similarity between calculated phonon-dispersion curves of h-BN and $2 \mathrm{D}-\mathrm{BN}$ is remarkable.

We also calculate the phonon-dispersion curves of 2D BN honeycomb structure by using PAW potentials ${ }^{21}$ as implemented in VASP (Ref. 23) for further checking of the results of our phonon calculation. Force constants are determined from the $(8 \times 8 \times 1)$ supercells. The phonon modes were calculated by using the direct method as implemented in the PHON (Ref. 44) software. The calculated phonon frequencies are almost identical with those calculated by DFPT method. In Fig. 3(d), we present the phonon density of states calculated for the $2 \mathrm{D} \mathrm{BN}$ honeycomb structure. Note that both calculations yield that vertical or out-of-plane acoustical (ZA) branch displaying parabolic dispersion gets negative frequencies as $k \rightarrow 0$.

Similar to BN atomic chains, this indicates structural instability as $\lambda \rightarrow \infty$. The ZA phonon branch of the 2D BN structure has negative frequencies within $\sim 1 / 10$ of the $\mathrm{BZ}$ around its center as shown Fig. 3. This indicates that for wave vectors $\mathbf{k}$ within this region of $\mathrm{BZ}$ the structure is unstable and hence the wavelengths $\lambda \gtrsim 10 a_{1}$ are not al-

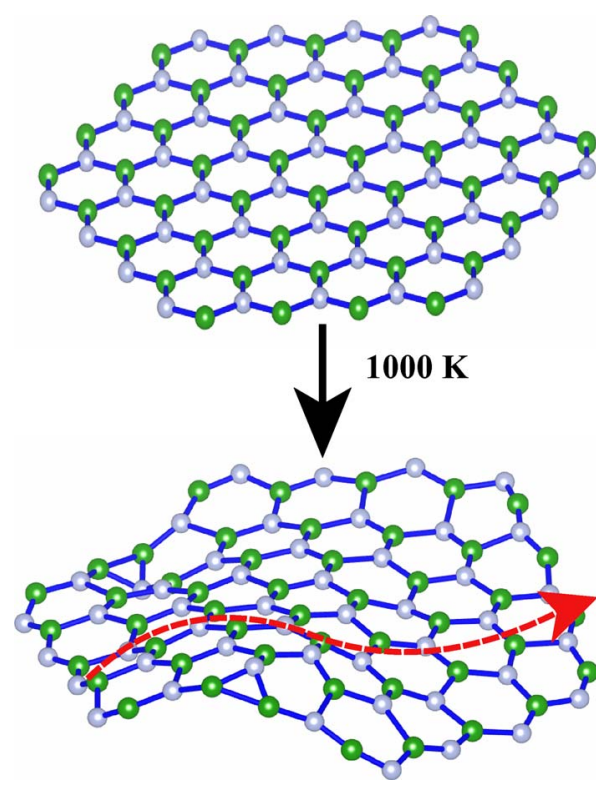

FIG. 4. (Color online) Upper: ideal structure of a hexagonal BN flake. Lower: atomic structure of the same flake after 200 time steps of first-principles MD calculations carried out at $1000 \mathrm{~K}$. The rippling of $2 \mathrm{D}$ structure is shown by the dashed arrow.

lowed. This implies the breakdown of the 2D periodicity in the corresponding lattice. Under these circumstances the long-range periodicity is destroyed by the rippling of the $2 \mathrm{D}$ lattice. In compliance with these arguments one expects that a finite-size flake of diameter $D \leqq 10 a_{1}$ can be stable since phonon modes with long wavelengths are not allowed. We carried out ab initio molecular-dynamics (MD) calculations at $T=1000 \mathrm{~K}$ on hexagonal finite-size BN flakes with time steps $\delta t=2 \times 10^{-15}$ s. As shown in Fig. 4, the structure of the flake was rippled but was not destroyed after 200 time steps.

\section{1D BN NANORIBBONS}

Similar to graphene, ${ }^{45}$ two unique orientations in the $2 \mathrm{D}$ $\mathrm{BN}$ yield nanoribbons with uniform edges. These are ABNNR and ZBNNR nanoribbons. The profile of the atomic configuration at both edges of the nanoribbon determines their electronic and magnetic properties. The properties can be modified by the passivation of the dangling bond of edge atoms by hydrogen. Because of their interesting electronic and spintronic properties, $\mathrm{BN}$ nanoribbons are attractive nanostructures for various device applications. Electronic properties of $\mathrm{BN}$ nanoribbons have been investigated in recent papers. ${ }^{16-18}$ The present study is complementary to previous studies.

\section{A. Electronic structure}

Here we present the results of our study on the electronic and magnetic properties of bare and hydrogen-passivated ABNNR and ZBNNRs. Bare and hydrogen passivation ABNNR are wide band-gap semiconductors. Similarly, hydrogen-passivated ZBNNRs are also semiconductor. The band gaps of these BN nanoribbons depend on the width of 


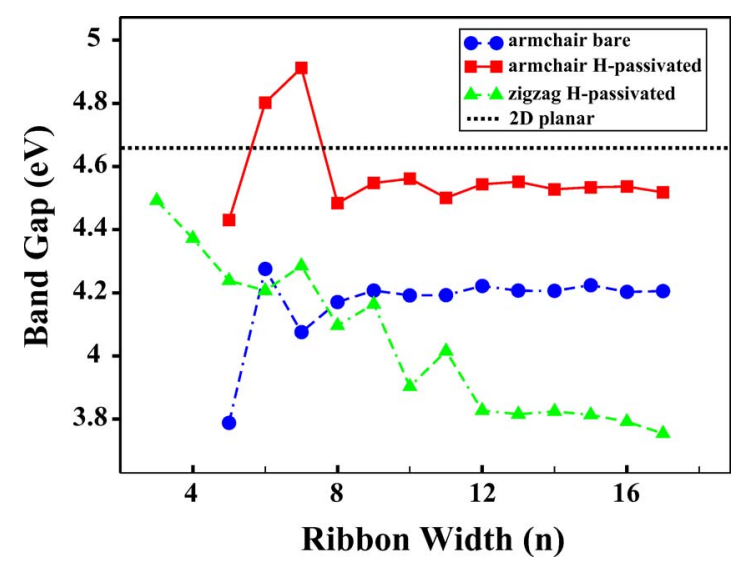

FIG. 5. (Color online) Energy band gap versus the width of the nanoribbons given in terms of the number of $\mathrm{B}-\mathrm{N}$ atom pairs in the primitive unit cell, $n$. Bare armchair nanoribbons ABNNR, hydrogen-passivated ABNNR, and hydrogen-passivated zigzag nanoribbons ZBNNR. Dotted line indicates the bulk band gap of 2D BN.

the nanoribbons $w$ or the numbers of $\mathrm{BN}$ pairs, $n$, in the primitive unit cell. The variation in the band gap $E_{G}$ as a function of $n$ is given in Fig. 5. Normally, the properties of nanoribbons approaches those of the $2 \mathrm{D}$ honeycomb structure as the width $n \rightarrow \infty$. However, due to the localized edge states the band gap of ZBNNR approaches a gap smaller than that of the $2 \mathrm{D} \mathrm{BN}$ honeycomb structure. ${ }^{17}$ For narrow $(n<8)$ bare and hydrogen-passivated ABNNRs the band gaps vary with $n$, but they are practically unaltered for $n$ $>8$. For $n>8$ the band gap of bare ABNNR is $0.4 \mathrm{eV}$ smaller than that of hydrogen-passivated ABNNR. The band gap of hydrogen-passivated ZBNNR is $4.5 \mathrm{eV}$ for $n=3$ but decrease to $3.8 \mathrm{eV}$ for $n=16$. However, its variation with $n$ is not monotonic for $5<n<13$; it rather displays familydependent oscillatory variation with changes as large as 0.4 $\mathrm{eV}$ between two consecutive values of $n$. On the other hand, bare ZBNNRs are found to be metallic.

The atomic and electronic structures of bare and hydrogen-passivated ABNNR are described in Fig. 6 for $n$ $=12$. The atoms at the edges of the bare ABNNR are reconstructed; while all boron atoms at the edge are lowered and adjacent nitrogen atoms are raised. The reconstruction pattern was not affected when the structure optimization is carried using double unit cells. Two bands of edge states occur just below the conduction band edge. These two bands are normally degenerate when $n$ is large, but the degeneracy is split when two edges start to couple. Filled bands of edge states occur $\sim-1 \mathrm{eV}$ below the top of the valance band edge. Normal states, on the other hand, have charge distributed uniformly in the ribbon. Because of the edge states the band gap is indirect and is $\sim 4.22 \mathrm{eV}$ wide. As reported earlier, ${ }^{17}$ band gaps of hydrogen-passivated ABNNRs converge to a value that is $0.02 \mathrm{eV}$ smaller than the band gap of 2D BN sheet. This difference is relatively higher $(\approx 0.1 \mathrm{eV})$ in our results. Furthermore our calculations reveal such a behavior for bare armchair nanoribbons. The band-gap value of bare nanoribbons converges to a value that is $\approx 0.4 \mathrm{eV}$ smaller than the $2 \mathrm{D}$ band gap. Upon passivation
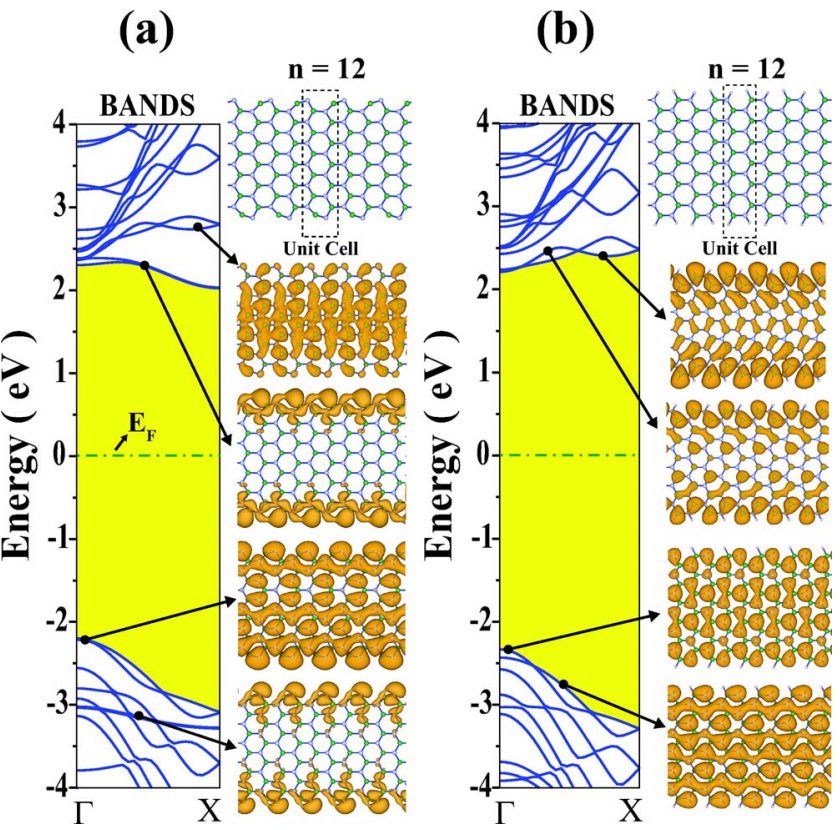

FIG. 6. (Color online) (a) Energy-band structure of bare armchair nanoribbon ABNNR having $n=12 \mathrm{~B}-\mathrm{N}$ pairs in the primitive unit cell. At the right-hand side of the bands, the schematic description of atomic structure with primitive unit cell delineated by dotted lines and isosurface charge distribution of specific states are shown. (b) Same as (a) but the dangling bonds at both edges are passivated by hydrogen atoms.

of the dangling bonds of $\mathrm{B}$ and $\mathrm{N}$ atoms situated at the edges with hydrogen atoms, these edge state bands are discarded from the band gap and reconstruction of edge atoms disappear. At the end, the band gap of H-passivated ABNNR becomes direct and increases by $\sim 0.3 \mathrm{eV}$.

The electronic and magnetic states of ZBNNR depend on whether their edges are passivated with hydrogen atoms. While a bare ZBNNR is magnetic and metallic, it becomes nonmagnetic and a wide band-gap semiconductor upon the passivation of $\mathrm{B}$ and $\mathrm{N}$ atoms at both edges. Moreover, its electronic and magnetic properties depend on whether only $\mathrm{B}$ or $\mathrm{N}$ side is passivated with hydrogen atoms. Accordingly, ZBNNRs provide us several alternatives for different electronic and magnetic properties. ${ }^{18}$ However, different magnetic states corresponding to different edge configuration, namely, bare or hydrogen passivated, are very sensitive to the parameters of the calculation. In Fig. 7 we present the calculated electronic structures of a ZBNNR with $n=6 \mathrm{~B}-\mathrm{N}$ pairs in a primitive unit cell for four different cases. These are both side free, only $\mathrm{N}$-side is passivated with hydrogen, only B-side is passivated with hydrogen, and both edges are passivated with hydrogen.

Bare ZBNNR having both edges that are free display different magnetic states (magnetic order), which are close in energy. Moreover, the ordering of these magnetic states with respect to their energy is sensitive to the criterion of energy convergence. To ensure the antiferromagnetic (AFM) order at edges, we considered double cells. The possible magnetic states are spin up, spin down for adjacent B atoms at one side and spin up, spin up for the adjacent $\mathrm{N}$ atoms at the other 
(a) BARE

(b) N-H

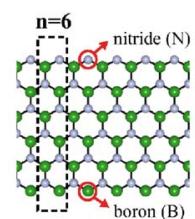

$\Delta p=\rho(\boldsymbol{t})-\rho(t)$
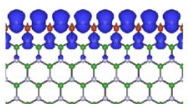

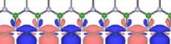

BANDS

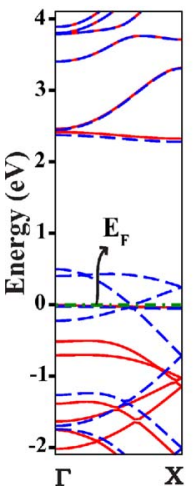

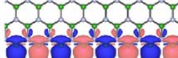

BANDS

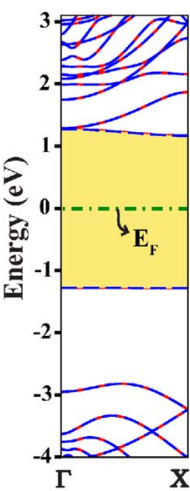

(c) B-H

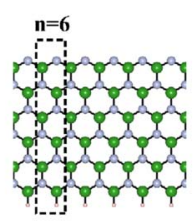

$\Delta \rho=\rho(t)-\rho(t)$
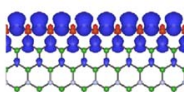

tomonen

(d) B-H, N-H
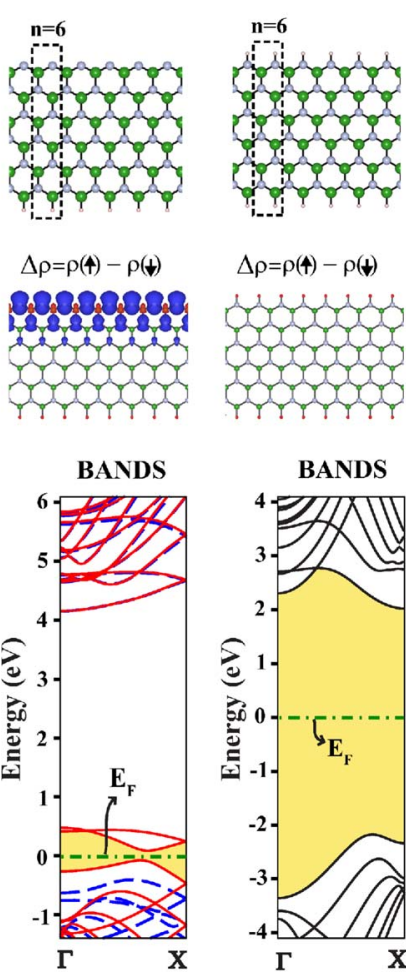

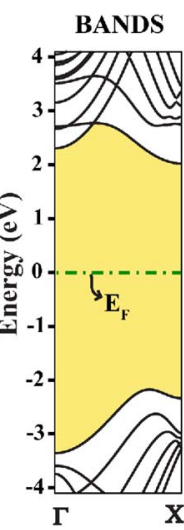

FIG. 7. (Color online) Top panels: atomic structures of ZBNNR. The primitive unit cell has $n=6 \mathrm{~B}-\mathrm{N}$ pairs delineated by dotted lines in the top panels. The unit cell is doubled due to the antiferromagnetic interaction between adjacent $\mathrm{N}$ atoms. Middle panels: isosurface plots of difference charge density between up-spin and downspin states, $\Delta \rho=\rho(\uparrow)-\rho(\downarrow)$. Bottom panels: energy-band structure with dotted (blue) and solid (red) lines showing spin-up and spindown states, respectively. (a) Bare ZBNNR; (b) B-side free, but $\mathrm{N}$-side is passivated by hydrogen atoms; (c) $\mathrm{N}$-side free, but $\mathrm{B}$-side is saturated by hydrogen atoms; (d) both sides are saturated by hydrogen atoms. The bands in (a)-(c) are calculated using double cell.

side, namely, $\uparrow \downarrow / \uparrow \uparrow$ spin configuration. Other possible spin configurations are $\uparrow \uparrow / \downarrow \downarrow, \uparrow \uparrow / \uparrow \uparrow, \uparrow \uparrow / \uparrow \downarrow$, and $\uparrow \downarrow / \uparrow \uparrow$. We found that the spin configuration, $\uparrow \downarrow / \uparrow \uparrow$ for ZBNNR having $12 \mathrm{~B}-\mathrm{N}$ pairs in a double unit cell, corresponds to the ground state. The other excited configurations, $\uparrow \uparrow / \downarrow \downarrow, \uparrow \uparrow / \uparrow \uparrow$, $\uparrow \uparrow / \uparrow \downarrow$, and $\uparrow \downarrow / \uparrow \downarrow$, have $6,7,35$, and $131 \mathrm{meV}$ higher total energies than the ground state. The orderings of these configurations are slightly different from that reported earlier. ${ }^{18}$ Although the reported ground-state configuration is the same as ours, excited-state orderings are different. For example, the $\uparrow \downarrow / \uparrow \downarrow$ state is the lowest energetic excited state in our case, while it is reported as the most energetic excited state in Barone and Peralta. ${ }^{18}$ This might be related with the methods used. Nevertheless, the difference between the earlier and present ground-state energies are within the accuracy limits of the DFT calculations. The ground-state spin configuration $\uparrow \downarrow / \uparrow \uparrow$ of the bare ZBNNR having both edges free is found to be a ferrimagnetic metal with $\mu=1.77 \mu_{B}$ per double cell, whereas the excited magnetic state with configuration $\uparrow \uparrow / \downarrow \downarrow$ is half metallic. Using the calculated energy difference between AFM and FM states we obtain a rough

estimate of the Curie temperature of ZBNNR as $T_{C}$ $\simeq 379 \mathrm{~K}$ for $n=6$.

In Fig. 7(b), ZBNNR with $\mathrm{N}$ edge passivated with hydrogen atoms is an AFM semiconductor. The AFM edge state is localized at the B side. When only the B side is passivated with hydrogen atoms the magnetic edge state is, this time, localized at the $\mathrm{N}$ side of the ribbon. As seen in Fig. 7(c) the ground state of ZBNNR is ferromagnetic with $\mu=2 \mu_{B}$ per double cell. Our calculations suggest that the nearestneighbor N-N interaction is ferromagnetic and the B-B interaction is antiferromagnetic. Finally, the ZBNNR becomes nonmagnetic when the atoms at both edges are passivated with hydrogen atoms. Earlier, Park and Louie ${ }^{17}$ studied hydrogen-passivated ABNNRs and ZBNNRs with widths of up to $10 \mathrm{~nm}$. Our results for hydrogen-passivated nanoribbons are in good agreement with their results, except that our results for zigzag ribbons obtained using GGA as well as LDA exhibit family-dependent oscillations for $5<n<13$.

\section{B. Elastic properties}

The elastic properties of BNNRs are examined through the variation in the total energy $E_{T}$ with respect to the applied uniaxial strain $\epsilon=\Delta c / c$, with $c$ being the lattice constant along the nanoribbon axis. Owing to ambiguities in defining the cross section of the ribbon one cannot determine Young's modulus rigorously. Instead we calculate $\kappa=\partial^{2} E_{T} / \partial c^{2}$ from the variation in $E_{T}$ to specify the elastic properties of quasi-1D nanoribbons. In Fig. 8(a) we show the variation in the total energy $E_{T}$ versus $\epsilon$. In order to lift the constraints imposed by periodic boundary conditions, calculations are performed for a supercell comprising five primitive unit cells having lattice constant $c_{s}=5 c$. For $\epsilon<0.10$, the variation in $E_{T}(\epsilon)$ is parabolic, and hence $\kappa$ is independent of $\epsilon$. For $\epsilon$ $>10$ the $E_{T}(\epsilon)$ curve deviates from parabola and becomes anharmonic. For higher values of strain in the plastic region, the ribbon undergoes structural transformation. For example, such a transformation occurred at $\epsilon=0.24$ with a sudden change in the $E_{T}(\epsilon)$ curve. The corresponding structure is illustrated as inset. The lattice constant $c_{s}$ increased from the initial value $21.5 \AA$ to $27.4 \AA$ corresponding to $\epsilon=0.27$.

In Fig. 8(b) $\kappa$ versus the width of the ribbon in terms of the number of B-N pair in the primitive unit cell $n$ is plotted for bare and hydrogen-passivated ABNNR. $\kappa(n)$ shows an approximately linear variation, indicating that the force constant is directly proportional to the width of the ribbon. One also sees that the strength of the ribbon increases upon passivation with hydrogen.

The behavior of bare and hydrogen-passivated ZBNNR under uniaxial tensile stress is similar to that of ABNNR. In Fig. 9 three regions, namely, elastic harmonic, elasticanharmonic, and plastic regions are seen. The sudden change in the $E_{T}(\epsilon)$ curve at $\epsilon \sim 0.23$ indicates a structural phase transformation, where the lattice constant $c_{s}$ elongates from the initial $\epsilon=0$ value of 19.8 to $25.7 \AA$ corresponding to $\epsilon$ $=0.3$. The structure of hydrogen-passivated ZBNNR before and after the structural transformation is shown as inset. Variation in $\kappa$ versus the ribbon width $n$ is calculated for bare and hydrogen-passivated ZBNNR shows an overall linear behavior as presented in Fig. 8(b). 
(a)

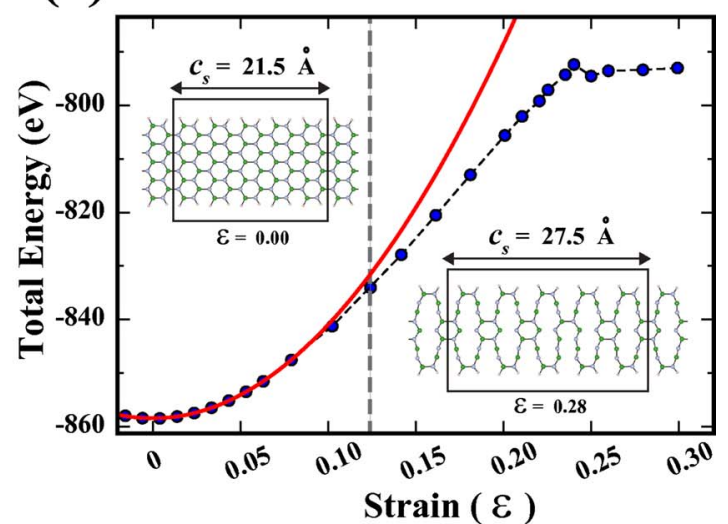

(b)

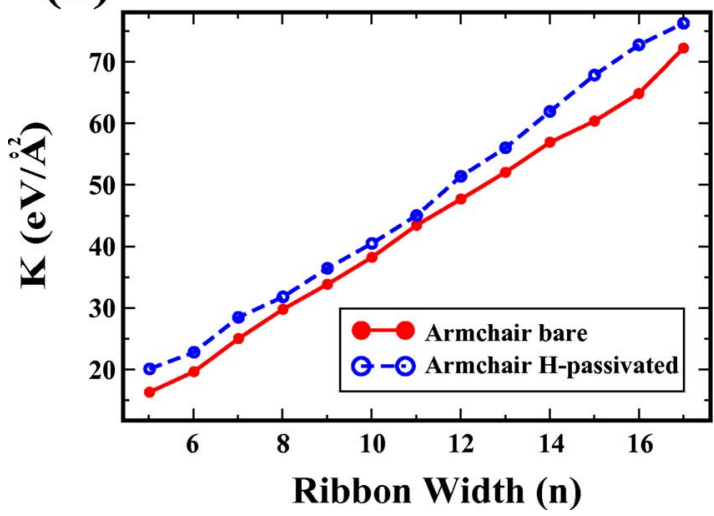

FIG. 8. (Color online) (a) Variation in the total energy of hydrogen-saturated ABNNR with strain $\epsilon$ is shown by the dashed curve with large black dots indicating the calculated data points $\left(c_{s}=5 c\right.$ and $\left.n=9\right)$. Harmonic, anharmonic, and plastic regions are distinguished. The harmonic part is fitted to a parabola presented by red-solid curve. Atomic structure shown by filled, empty, and very small empty circles represent B, N, and H atoms. Supercell comprising five primitive unit cells are shown in the harmonic and plastic regions. (b) Variation in $\kappa=\partial^{2} E_{T} / \partial c^{2}$ versus the ribbon width $n$ calculated for bare and hydrogen-passivated ABNNR.

\section{Vacancy and antisite defects}

It is known that the vacancy defect in $2 \mathrm{D}$ graphene ${ }^{46-50}$ and graphene nanoribbons ${ }^{51,52}$ gives rise to crucial changes in the electronic and magnetic structures. According to Lieb's theorem, ${ }^{53}$ the net magnetic moment per cell is determined with the difference in the number of atoms belonging to different sublattices, i.e., $\mu=\left(N_{\mathrm{B}}-N_{\mathrm{N}}\right) \mu_{\mathrm{B}}$. While DFT calculations on vacancies in $2 \mathrm{D}$ graphene and armchair graphene nanoribbons confirmed Lieb's theorem, results are diversified for vacancies in zigzag graphene nanoribbons. ${ }^{51,52}$ Therefore, the effect of vacancy defects on the properties of BNNRs is of interest.

Earlier activation energies and reaction paths for diffusion and nucleation monovacancy and divacancy in h-BN layers have been investigated by using density-functional tightbinding method. The formation energies were calculated to be 11.22 and $8.91 \mathrm{eV}$, for $\mathrm{B}$ and $\mathrm{N}$ vacancies, respectively. ${ }^{54}$ The possible magnetism induced by nonmagnetic impurities and vacancy defects in a $\mathrm{BN}$ sheet has been investigated

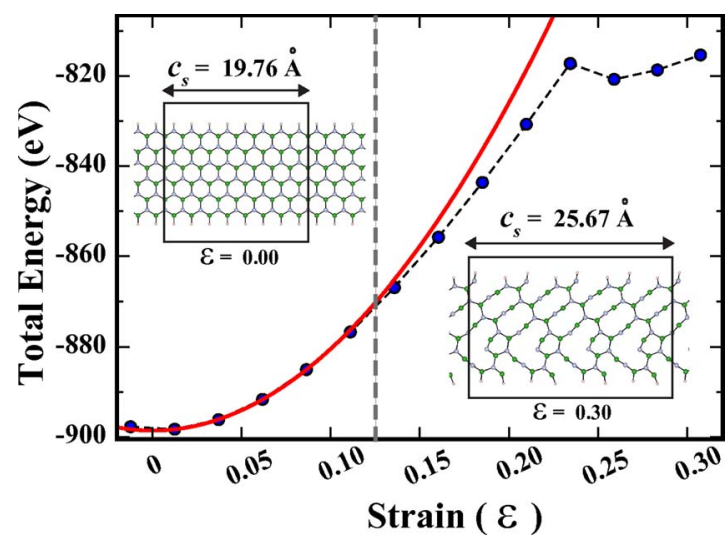

FIG. 9. (Color online) Variation in the total energy of hydrogensaturated ZBNNR shown by the dashed curve with large black dots indicating the calculated data points. Harmonic, anharmonic, and plastic regions are distinguished. The harmonic part is fitted to a parabola presented by a red-solid curve. Atomic structure of the ribbon in a supercell comprising eight unit cells $\left(c_{s}=8 c\right.$ and $\left.n=6\right)$ are shown before and after structural transformation as inset.

from the first principles. The magnetic moment associated by nonmagnetic atoms substituting $\mathrm{B}$ or $\mathrm{N}$ has been calculated to be $1 \mu_{B} \cdot{ }^{55}$ Based on first-principles calculations, the magnetic moment of a $\mathrm{N}$ vacancy in a $2 \mathrm{D} \mathrm{BN}$ sheet has been predicted to be $1 \mu_{B}$. In the case of a B vacancy, three neighboring $\mathrm{N}$ atoms are displaced further apart from each other and the net magnetic moment is predicted to be $3 \mu_{B} \cdot{ }^{56} \mathrm{An}$ other calculation of defects in a BN monolayer found that three dangling bonds associated with a B vacancy lead to total spin $S=3 / 2$, i.e., $3 \mu_{B}{ }^{57}$

The effects of vacancies of $\mathrm{BN}$ nanoribbons have not been treated yet. Here we investigate the effect of $\mathrm{B}, \mathrm{N}, \mathrm{B}+\mathrm{N}$ divacancy, and $\mathrm{B}+\mathrm{N}$ antisite on the electronic and magnetic properties of ABNNR and ZBNNR. Within periodic boundary conditions, a vacancy defect in an ABNNR of width $n$ $=12$ is repeated in every fifth primitive unit cell to yield minute defect-defect coupling. As shown in Fig. 10(a) ABNNR with B vacancy becomes ferromagnetic with a net magnetic moment of $\mu=1 \mu_{B}$ per unit cell. Similarly, a $\mathrm{N}$ vacancy gives rise to a net magnetic moment of $\mu=1 \mu_{B}$ per unit cell. ABNNRs having either periodic $\mathrm{B}+\mathrm{N}$ divacancy or antisite defect for every five unit cell remain nonmagnetic. The calculated values of magnetic moments are in compliance with Lieb's theorem. We found that the structural relaxation is crucial to obtain correct values of magnetic moments. In particular, initially we calculated $\mu=3 \mu_{B}$ for the relaxed structure of the $\mathrm{B}$ vacancy. However, the neighboring $\mathrm{N}$ atoms distorted slightly from their equilibrium, the structure is relaxed further and had lowered the total energy. As a result, the magnetic moment was calculated as $\mu=1 \mu_{B}$. The energy-band structures in Figs. 10(a)-10(d) are calculated for periodic vacancy defects repeating in every four primitive cell. The Fermi levels are assigned according to the occupancy of vacancy states. We note that the empty state associated with the B vacancy in Fig. 10 is holelike. The states associated with the $\mathrm{N}$ vacancy that occur near the edge of the conduction band are donorlike.

The situation with ZBNNR is similar to that in ABNNR discussed above since hydrogen-passivated ZBNNR is non- 
(a)

(b)

(c)

(d)

A-BNNR+1BV A-BNNR+1NV A-BNNR+1B,1NV:A-BNNR+antisite $\mu=1.00 \mu_{\mathrm{B}} \quad \mu=1.00 \mu_{\mathrm{B}} \quad \mu=0 \mu_{\mathrm{B}} \quad \mu=0 \mu_{\mathrm{B}}$

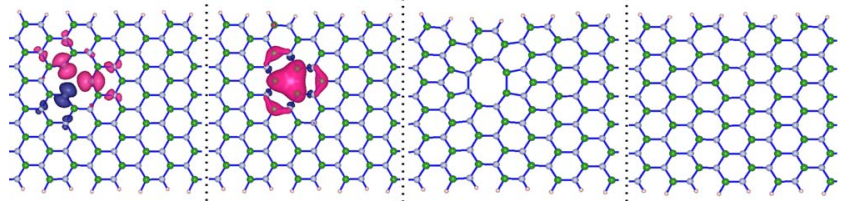

BANDS

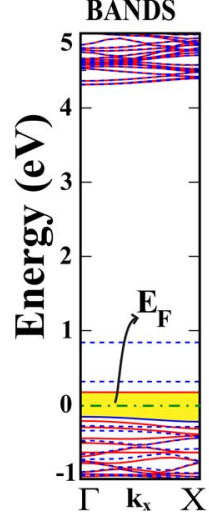

BANDS
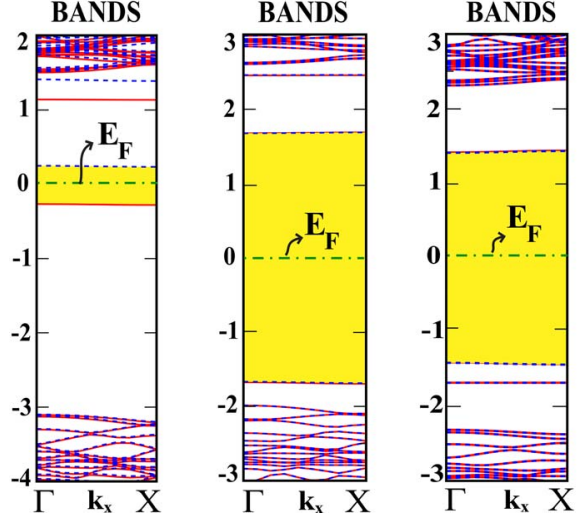

FIG. 10. (Color online) Relaxed atomic structures and corresponding energy bands of hydrogen-passivated ABNNR with $n$ $=12$ having a point defect located periodically in every four primitive cell. Blue filled, empty, and small circles represent $\mathrm{B}, \mathrm{N}$, and $\mathrm{H}$ atoms, respectively. Pink/light and blue/dark isosurface plots are for spin-up and spin-down states. (a) Single B vacancy; (b) single N vacancy; (c) B-N divacancy; (d) antisite defect.

magnetic as ABNNR. As shown in Fig. 11, a periodic B or $\mathrm{N}$ vacancy repeated in every eight unit cell of hydrogensaturated ZBNNR with $n=6$ has a net magnetic moment of $\mu=1 \mu_{B}$ per supercell. ZBNNR passivated with hydrogen atoms at both edges and having either periodic $\mathrm{B}+\mathrm{N}$ divacancy or antisite defect repeating in every eight unit cell is nonmagnetic. The type of the periodic vacancy defect modifies the band gap of ZBNNR from 4.2 to $2.21 \mathrm{eV}$ for divacancy but to $2.8 \mathrm{eV}$ for antisite. The calculated magnetic moment of hydrogen-passivated ZBNNR is in agreement with Lieb's theorem. We note that in zigzag graphene nanoribbons magnetic edge states survive even after hydrogen passivation and interact with the magnetic moments of vacancies. ${ }^{52}$ This interaction causes deviation from the prediction of Lieb's theorem.

\section{DISCUSSION AND CONCLUSIONS}

In various allotropic forms of $\mathrm{BN}$ the dimensionality play a crucial role. For the sake of comparison, we present the calculated values of $\mathrm{BN}$ for different allotropic forms of $\mathrm{BN}$ in different dimensionalities. One sees that the B-N double bond of 1D BN atomic chain is shortest and is $1.31 \AA$. The $\mathrm{s} p^{2}$ bond of h-BN and 2D BN has an intermediate value of $1.45 \AA$. Therefore, h-BN can be considered as quasi-twodimensional. Three-dimensional wurtzite and zinc-blende BN crystal have $\mathrm{s} p^{3}$ bonding with $d=1.56 \AA$, which is largest among the allotropic forms studied here. According to (a)

Z-BNNR +1BV

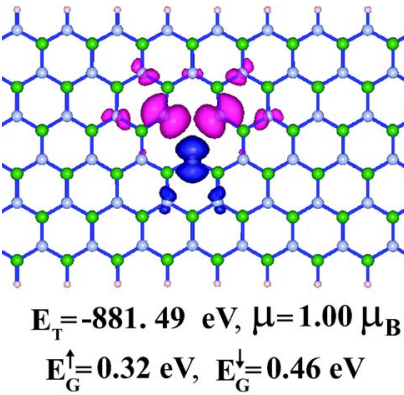

(c)

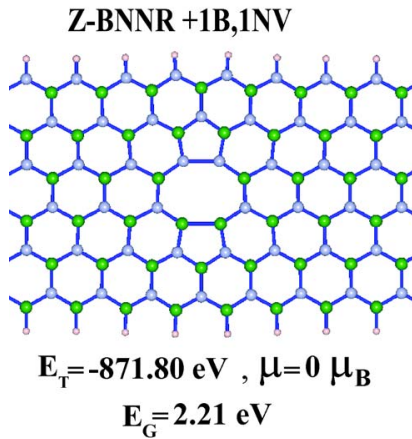

(b)

Z-BNNR +1NV

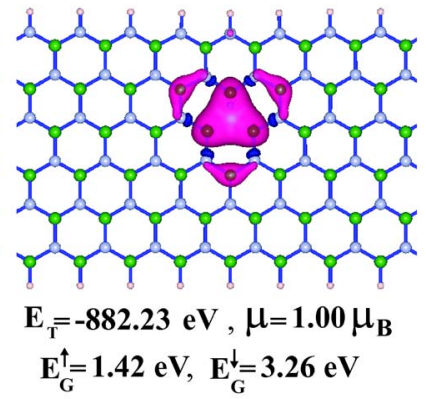

(d)

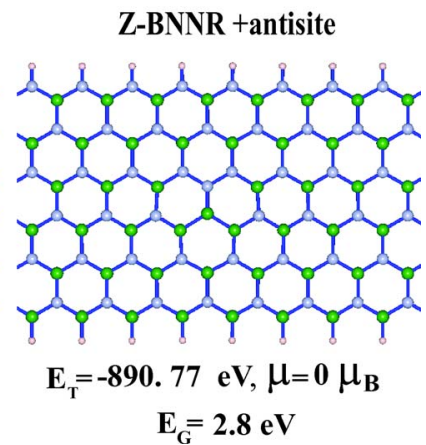

FIG. 11. (Color online) Relaxed atomic structures of hydrogenpassivated ZBNNR with $n=6$ having a vacancy defect located periodically in every eight primitive cell $\left(c_{s} \approx 8 c\right.$ and $\left.n=6\right)$. Filled, empty, and small circles represent $\mathrm{B}, \mathrm{N}$, and $\mathrm{H}$ atoms, respectively. Pink and blue isosurface plots are spin-up and spin-down states, respectively. (a) Single B vacancy; (b) single N vacancy; (c) B $+\mathrm{N}$ divacancy; (d) antisite defect.

GGA results the cohesive energy of $2 \mathrm{D} \mathrm{BN}$ is $3 \mathrm{meV}$ larger than that of h-BN. This is due to the fact that the GGA calculation cannot account the van der Waals interaction between atomic layers of h-BN. However, the calculations using LDA, where the van der Waals interactions are better accounted, yield the cohesive energy of h-BN which is $\sim 57 \mathrm{meV}$ larger than that of the $2 \mathrm{D} \mathrm{BN}$ as one expects. The charge transfer $\Delta Q$ from $\mathrm{B}$ to $\mathrm{N}$ atom increases with decreasing dimensionality. This is due to fact that $d$ decreases with decreasing dimensionality. The coordination number increases with increasing dimension.

In $2 \mathrm{D} \mathrm{BN}$ honeycomb structures and in its zigzag and armchair nanoribbons, the B-N bond formed from the bonding $\mathrm{s} p^{2}$ hybrid orbitals from $\mathrm{B}$ and $\mathrm{N}$ atoms is essential. Owing to the transfer of charge from $\mathrm{B}$ to $\mathrm{N}$ the $\mathrm{B}-\mathrm{N}$ bond acquires an ionic character, which underlies the semiconducting properties with wide band gap.

Bare armchair nanoribbon of $2 \mathrm{D} \mathrm{BN}$ is again a nonmagnetic wide band-gap semiconductor, the band gap of which is practically unaltered with width $n>8$. Upon passivation with hydrogen, band gap of the ribbon increases by $0.3 \mathrm{eV}$. As for zigzag nanoribbons, they provide a number of interesting properties. When its both edges are bare, it is ferromagnetic metal. When its $\mathrm{N}$ edge is passivated with hydrogen, it becomes an antiferromagnetic semiconductor. In the reverse case, namely, when the B side is passivated, it becomes a 
ferromagnetic semiconductor. When both edges are passivated, it becomes a nonmagnetic wide band-gap semiconductor. The band gap as well as the magnetic state of a ribbon can be modified by periodic vacancy defects. Finally, $\mathrm{BN}$ nanoribbons have been found to be strong, quasi-onedimensional, and stable structures. They can sustain up to high strains, and they stretch in the plastic region with structural transformations.

Briefly, the calculated electronic, magnetic, and mechanical properties of 2D BN honeycomb structure and its nanoribbons present interesting but some differences from graphene. In this respect $\mathrm{BN}$ honeycomb structure and its nanoribbons are complimentary to graphene. The properties of 2D BN honeycomb structure can be changed upon functionalization with foreign atoms. Interesting quantum structures (such as single and series quantum dots, resonant tunneling double barriers, and multiple quantum well structures) based on heterostructures and core shell structures of lattice matched graphene and $\mathrm{BN}$ can be formed, since the band gap of $\mathrm{BN}$ is much larger than that of graphene.

\section{ACKNOWLEDGMENTS}

Part of the computational resources has been provided by UYBHM at Istanbul Technical University through Grant No. 2-024-2007. *ciraci@fen.bilkent.edu.tr

${ }^{1}$ K. S. Novoselov, A. K. Geim, S. V. Morozov, D. Jiang, Y. Zhang, S. V. Dubonos, I. V. Grigorieva, and A. A. Firsov, Science 306, 666 (2004).

${ }^{2}$ Y. Zhang, Y.-W. Tan, H. L. Stormer, and P. Kim, Nature (London) 438, 201 (2005).

${ }^{3}$ C. Berger, Z. Song, X. Li, X. Wu, N. Brown, C. Naud, D. Mayou, T. Li, J. Hass, A. N. Marchenkov, E. H. Conrad, P. N. First, and W. A. de Heer, Science 312, 1191 (2006).

${ }^{4}$ A. K. Geim and K. S. Novoselov, Nature Mater. 6, 183 (2007).

${ }^{5}$ Y.-W. Son, M. L. Cohen, and S. G. Louie, Nature (London) 444, 347 (2006).

${ }^{6}$ H. Sevinçli, M. Topsakal, E. Durgun, and S. Ciraci, Phys. Rev. B 77, 195434 (2008).

${ }^{7}$ M. Topsakal, H. Sevinçli, and S. Ciraci, Appl. Phys. Lett. 92, 173118 (2008).

${ }^{8}$ K. S. Novoselov, D. Jiang, F. Schedin, T. Booth, V. V. Khotkevich, S. Morozov, and A. K. Geim, Proc. Natl. Acad. Sci. U.S.A. 102, 10451 (2005).

${ }^{9}$ D. Pacilé, J. C. Meyer, Ç. Ö. Girit, and A. Zettl, Appl. Phys. Lett. 92, 133107 (2008).

${ }^{10}$ A. Nagashima, N. Tejima, Y. Gamou, T. Kawai, and C. Oshima, Phys. Rev. Lett. 75, 3918 (1995).

${ }^{11}$ L. Bourgeois, Y. Bando, W. Q. Han, and T. Sato, Phys. Rev. B 61, 7686 (2000).

${ }^{12}$ N. G. Chopra, R. J. Luyken, K. Cherrey, V. H. Crespi, M. L. Cohen, S. G. Louie, and A. Zettl, Science 269, 966 (1995).

${ }^{13}$ C. Zhi, Y. Bando, C. Tang, and D. Golberg, Appl. Phys. Lett. 87, 063107 (2005).

${ }^{14}$ D. Golberg, A. Rode, Y. Bando, M. Mitome, E. Gamaly, and B. Luther-Davies, Diamond Relat. Mater. 12, 1269 (2003).

${ }^{15}$ Y. J. Chen, H. Z. Zhang, and Y. Chen, Nanotechnology 17, 786 (2006).

${ }^{16}$ Z. Zhang and W. Guo, Phys. Rev. B 77, 075403 (2008).

${ }^{17}$ Cheol-Hwan Park and Steven G. Louie, Nano Lett. 8, 2200 (2008).

${ }^{18}$ V. Barone and J. E. Peralta, Nano Lett. 8, 2210 (2008).

${ }^{19}$ X. Li, L. Zhang, S. Lee, and H. Dai, Science 319, 1229 (2008).

${ }^{20}$ X. Wang, Y. Ouyang, X. Li, H. Wang, J. Guo, and H. Dai, Phys. Rev. Lett. 100, 206803 (2008).

${ }^{21}$ P. E. Blöchl, Phys. Rev. B 50, 17953 (1994).

${ }^{22}$ J. P. Perdew, J. A. Chevary, S. H. Vosko, K. A. Jackson, M. R.
Pederson, D. J. Singh, and C. Fiolhais, Phys. Rev. B 46, 6671 (1992).

${ }^{23}$ G. Kresse and J. Hafner, Phys. Rev. B 47, 558 (1993).

${ }^{24}$ G. Kresse and J. Furthmüller, Phys. Rev. B 54, 11169 (1996).

${ }^{25}$ S. Baroni, A. Del Corso, S. Girancoli, and P. Giannozzi, http:/ www.pwscf.org/

${ }^{26}$ A. Catellani, M. Posternak, A. Baldereschi, and A. J. Freeman, Phys. Rev. B 36, 6105 (1987).

${ }^{27}$ L. Liu, Y. P. Feng, and Z. X. Shen, Phys. Rev. B 68, 104102 (2003).

${ }^{28}$ N. Ooi, V. Rajan, J. Gottlieb, Y. Catherine, and J. B. Adams, Modell. Simul. Mater. Sci. Eng. 14, 515 (2006).

${ }^{29}$ K. Shimada, T. Sota, and K. Suzuki, J. Appl. Phys. 84, 4951 (1998).

${ }^{30}$ M. P. Surh, S. G. Louie, and M. L. Cohen, Phys. Rev. B 43, 9126 (1991).

${ }^{31}$ R. T. Senger, S. Tongay, E. Durgun, and S. Ciraci, Phys. Rev. B 72, 075419 (2005).

${ }^{32}$ A. V. Kurdyumov, V. L. Solozhenko, and W. B. Zelyavski, J. Appl. Crystallogr. 28, 540 (1995).

${ }^{33}$ Caution has to be taken in using the values of band gap calculated with DFT as in the present study, since the value of band gap is usually underestimated by DFT.

${ }^{34}$ The calculations of $\Delta Q$ have been carried out by using the PWSCF software (Ref. 25). It should be noted that the ground-state properties do not differ no matter when lattice constants and atomic configurations are optimized using either VASP or PWSCF.

${ }^{35}$ S. Tongay, R. T. Senger, S. Dag, and S. Ciraci, Phys. Rev. Lett. 93, 136404 (2004).

${ }^{36}$ E. Rokuta, Y. Hasegawa, K. Suzuki, Y. Gamou, C. Oshima, and A. Nagashima, Phys. Rev. Lett. 79, 4609 (1997).

${ }^{37}$ L. Wirtz, A. Rubio, R. A. delaConcha, and A. Loiseau, Phys. Rev. B 68, 045425 (2003).

${ }^{38}$ G. Kern, G. Kresse, and J. Hafner, Phys. Rev. B 59, 8551 (1999).

${ }^{39}$ W. J. Yu, W. M. Lau, S. P. Chan, Z. F. Liu, and Q. Q. Zheng, Phys. Rev. B 67, 014108 (2003).

${ }^{40}$ J. Serrano, A. Bosak, R. Arenal, M. Krisch, K. Watanabe, T. Taniguchi, H. Kanda, A. Rubio, and L. Wirtz, Phys. Rev. Lett. 98, 095503 (2007).

${ }^{41}$ V. L. Solozhenko, G. Will, and F. Elf, Solid State Commun. 96, 1 (1995). 
${ }^{42}$ Y. Miyamoto, M. L. Cohen, and S. G. Louie, Phys. Rev. B 52, 14971 (1995).

${ }^{43}$ F. Liu, P. Ming, and J. Li, Phys. Rev. B 76, 064120 (2007).

${ }^{44}$ D. Alfè, http://chianti.geol.ucl.ac.uk/dario

${ }^{45}$ V. Barone, O. Hod, and G. E. Scuseria, Nano Lett. 6, 2748 (2006).

${ }^{46}$ P. Esquinazi, D. Spemann, R. Höhne, A. Setzer, K.-H. Han, and T. Butz, Phys. Rev. Lett. 91, 227201 (2003).

${ }^{47}$ A. Hashimoto, K. Suenaga, A. Gloter, K. Urita, and S. Iijima, Nature (London) 430, 870 (2004).

${ }^{48}$ O. V. Yazyev and L. Helm, Phys. Rev. B 75, 125408 (2007).

${ }^{49}$ M. A. H. Vozmediano, M. P. Lopez-Sancho, T. Stauber, and F. Guinea, Phys. Rev. B 72, 155121 (2005).
${ }^{50}$ L. Brey, H. A. Fertig, and S. Das Sarma, Phys. Rev. Lett. 99, 116802 (2007).

${ }^{51}$ J. J. Palacios, J. Fernandez-Rossier, and L. Brey, Phys. Rev. B 77, 195428 (2008).

${ }^{52}$ M. Topsakal, E. Aktürk, H. Sevinçli, and S. Ciraci, Phys. Rev. B 78, 235435 (2008).

${ }^{53}$ E. H. Lieb, Phys. Rev. Lett. 62, 1201 (1989).

${ }^{54}$ A. Zobelli, C. P. Ewels, A. Gloter, and G. Seifert, Phys. Rev. B 75, 094104 (2007).

${ }^{55}$ R. F. Liu and C. Cheng, Phys. Rev. B 76, 014405 (2007).

${ }^{56}$ M. S. Si and D. S. Xue, Phys. Rev. B 75, 193409 (2007).

${ }^{57}$ S. Azevedo, J. R. Kashny, C. M. C. de Castilho, and F. De Brito, Nanotechnology 18, 495707 (2007). 